\title{
Heat Dissipation of Hybrid Iron Oxide-Gold Nanoparticles in an Agar Phantom
}

\author{
Anthony Curtis ${ }^{1}$, Maryam Malekigorji' ${ }^{1}$ Joseph Holman ${ }^{2}$, Mark Skidmore ${ }^{2}$ and Clare Hoskins ${ }^{1 *}$ \\ ${ }^{1}$ Institute for Science and Technology in Medicine, Faculty of Health, Keele University, Keele, ST5 5BG, UK \\ ${ }^{2}$ School of Life Sciences, Faculty of Natural Sciences, Keele University, Keele, ST5 5BG, UK
}

\begin{abstract}
Hybrid iron oxide-gold nanoparticles (HNPs) have shown potential in cancer therapy as agents for tumour ablation and thermal switches for targeted drug release. Heat generation occurs by exploitation of the surface plasmon resonance of the gold coating, which usually occurs at the maximum UV absorption wavelength. However, lasers at such wavelength are often expensive and highly specialised. Here, we report the heating and monitoring of heat dissipation of HNPs suspended in agar phantoms using a relatively inexpensive $\mathrm{Ng}$ : YAG pulsed $1064 \mathrm{~nm}$ laser source. The particles experience heating of up to $40^{\circ} \mathrm{C}$ with a total area of heat dissipation up to $132.73 \mathrm{~mm}^{2}$ from the $1 \mathrm{~mm}$ diameter irradiation point after 60 seconds. This work reports the potential and possible drawbacks of these particles for translation into cancer therapy based on our findings.
\end{abstract}

Keywords: Hybrid nanoparticles; Surface plasmon resonance; Heat dissipation; Thermo-responsive drug delivery; Laser irradiation

\section{Introduction}

Advances in technology have led to the exploitation of inorganic molecules in cancer therapy. These include nanoparticles composed of iron oxide [1], gold [2], quantum dots [3], carbon nanotubes [4], silver nanoparticles [5] and many more. Hybrid nanoparticles (HNPs) composed of an iron oxide core surrounded by a gold shell have shown potential for image guided cancer therapy [6]. This is due to the unique properties attributed from their architecture. The iron oxide core confers magnetic ability whilst the gold shell increases biocompatibility and offers further potential as a triggered heat source. When gold nanoparticles or shells are irradiated with a unique wavelength they undergo surface plasmon resonance (SPR) [7]. This phenomenon arises when the light becomes scattered and absorbed by the particles, the absorbed light rapidly transfers the light energy into heat energy and hence the ability to act as a localized heating source is possessed [8]. The exploitation of SPR has been reported in many publications in relation to both tumour ablation [9] and thermally triggered drug delivery [10].

In cancer therapy tumour ablation is an exciting alternative to chemotherapy. Often traditional chemotherapies cannot penetrate the dense tumor vasculature and drug resistance can also occur resulting in low efficiency of treatment and poor patient prognosis [11]. Tumour ablation is the process of destroying the tissue completely without the possibility of recovery [12]. This is carried out by heating the tissue to a temperature above the limit of cell survival which is thought to be $50^{\circ} \mathrm{C}$ [13]. Thus the cells are effectively 'burned'. This process is currently carried out using laser irradiation at a wavelength of $532 \mathrm{~nm}$ [14]. At this wavelength when the laser light encounters body tissue it is absorbed resulting in heating. However, this process is indiscriminate and healthy tissues are affected along with cancerous tissue. Development of gold nanoparticles and nano-shells has allowed for advances in this technology whereby, the wavelength can be increased into a region where safe tissue penetration can occur and heating is only experienced by the nanoparticulates in a highly localised manner.

Thermo-responsive drug delivery systems using gold nanoparticles or nano-shells also exploit the SPR effect [15]. Here the particles are heated in a controlled manner with specified laser power and duration to reach an optimal temperature. This temperature should exceed the normal body temperature for healthy tissue and tumour tissue but also be less than the kill temperature of the cells. The temperature rise in thermo-responsive delivery triggers release of the cargo payload from the nanoparticles into the surrounding vasculature or intracellular environment. The advantage of these systems is the ability to increase the drug permeation into the dense tumours, which increases efficacy. It is well documented that once encapsulated or conjugated onto nanoparticulates, cellular uptake is increased possibly due to the activation of endocytosis [16]. Additionally, nanoparticles have been shown to passively target tumour tissue via the enhanced permeability and retention effect. This phenomenon is due to accumulation of these nano-systems inside the poorly formed capillaries of the tumour vasculature, which cannot be accessed by larger scale molecules [17]. The poor lymphatic drainage in these areas results in vast accumulation, which can act as a targeting mechanism for cancer therapies. This can encourage deeper drug penetration in dense stroma, an efficacylimiting step in many cancer therapies.

In order for HNPs to be clinically relevant, an in-depth knowledge of heat dissipation is required in order to properly understand depth of heat penetration experienced and the effect of this heating on surrounding tissues. Work has been reported for gold nanoparticles and nano-shells, however many of these studies are mathematical models and not based on actual experiments as well as using laser wavelengths deemed to be inappropriate for this clinical application $[18,19]$. Clinically, the optimal wavelengths for laser irradiation of nanoparticles are within the 'biological near infrared region (NIR)' which is between $650 \mathrm{~nm}$ to $1350 \mathrm{~nm}$ [20]. Laser beams inside the

${ }^{*}$ Corresponding author: Clare Hoskins, Institute for Science and Technology in Medicine, Faculty of Health, Keele University, Keele, ST5 5BG, UK, Tel: +441782 734799; E-mail: c.hoskins@keele.ac.uk

Received October 01, 2015; Accepted November 02, 2015; Published November 10, 2015

Citation: Curtis A, Malekigorji M, Holman J, Skidmore M, Hoskins C (2015) Heat Dissipation of Hybrid Iron Oxide-Gold Nanoparticles in an Agar Phantom. Nanomed Nanotechnol 6: 335. doi:10.4172/2157-7439.1000335

Copyright: $\odot 2015$ Curtis A, et al. This is an open-access article distributed under the terms of the Creative Commons Attribution License, which permits unrestricted use, distribution, and reproduction in any medium, provided the original author and source are credited. 
NIR window are capable of deep tissue penetration due to the high transmisivity of water and haemoglobin within these wavelengths [21]. Many of the reports to date have been carried out using a $532 \mathrm{~nm}$ green light source. As mentioned previously, light of this wavelength unfortunately does not pass through biological tissues. Instead it becomes absorbed and this results in heat discharge of nanoparticles as well as the biological tissue rather than sole heating of nanoparticles. Hence, different wavelengths need to be explored in order for thermoresponsive delivery using HNPs to be clinically relevant.

The optimal wavelength for SPR irradiation usually occurs between $650-750 \mathrm{~nm}$ for gold nanoparticles and nano-shells, though this can be further increased upon surface functionalisation [22]. Figure 1 shows a representative UV spectrum of a gold nanoparticle. As seen in the spectra, although the optimal wavelength sits around $670 \mathrm{~nm}$ the peak is very broad and covers a large range of wavelengths. Thus, irradiation at $532 \mathrm{~nm}$ as shown in other studies results in heating. This is also true of irradiation at the red end of the spectra at $1064 \mathrm{~nm}$. Here a heating effect will be observed, this will have decreased efficiency compared with the lambda $\max \left(\lambda_{\max }\right)$, however, there is still potential. Whilst it is advantageous for this therapy to use a wavelength at the $\lambda_{\max }$, laser sources at such wavelengths are very expensive and would therefore form another hurdle before clinical acceptance was reached.

Laser systems using $1064 \mathrm{~nm}$ pulsed sources are widely used both in clinical settings for ophthalmic treatment and also in the cosmetic industry for hair removal and tattoo removal [23]. Thus, these systems are relatively cheap, portable and penetrate biological tissues with no adverse effect. Other studies regarding the heating of nanoparticles via SPR exploitation have shown that using pulsed laser irradiation is more effective than continuous laser irradiation [24]. Findings show that the higher energy in the short pulses result in greater thermal output and hence is preferable.

In this study we aim to investigate the heating potential and dissipation of HNPs after irradiation using a pulsed laser source at $1064 \mathrm{~nm}$. The HNPs were suspended in an agar phantom to mimic biological tissue, two methods of measurement of thermal change will be used and compared for accuracy. The effect of particle concentration on thermal change and resultant cooling will be investigated. It is proposed that these studies will be useful in determining whether our HNPs can be used in future studies as localised heaters for either thermal ablation or thermo-responsive drug delivery with $1064 \mathrm{~nm}$ irradiation.

\section{Materials and Methods}

All reagents were purchased from SigmaAldrich, UK unless otherwise stated. All optical components were purchased from Thor Labs, Germany.

\section{Synthesis of hybrid nanoparticles}

HNPs were synthesised and characterised as previously reported [25]. Briefly, iron oxide $\left(\mathrm{Fe}_{3} \mathrm{O}_{4}\right)$ nanoparticles were characterized using a simple precipitation reaction: $\mathrm{NaOH}(1.030 \mathrm{~g})$ and $\mathrm{KNO}_{3}(1.820 \mathrm{~g})$ in deionised water $\left(\mathrm{H}_{2} \mathrm{O}, 180 \mathrm{~mL}\right)$ was refluxed under nitrogen $\left(\mathrm{N}_{2}\right)$ for $1 \mathrm{~h}$. $\mathrm{Fe}_{3} \mathrm{O}_{4} \cdot 7 \mathrm{H}_{2} \mathrm{O}(3.89 \mathrm{~g})$ in $\mathrm{H}_{2} \mathrm{SO}_{4}(20 \mathrm{~mL})$ was added to the reaction and heated for $24 \mathrm{~h}$ at $90^{\circ} \mathrm{C}$. The black precipitate was cooled on ice and washed in $\mathrm{H}_{2} \mathrm{O}$. A poly(ethylenimine) (PEI) intermediate was added via probe sonication of the particles $\left(5 \mathrm{~mL}\right.$ with $\left.5 \mathrm{mg} / \mathrm{mL}^{-1}\right)$ polymer in deionised water rendering $\mathrm{Fe}_{3} \mathrm{O}_{4}$-PEI. Gold seeds $(2 \mathrm{~nm})$ were attached to the PEI surface $\left(\mathrm{Fe}_{3} \mathrm{O}_{4}\right.$-PEI- $\left.\mathrm{Au}_{\text {seed }}\right)$ followed by reduction of $\mathrm{HAuCl}_{4}$ forming a complete shell [26]. The HNPs were washed extensively with deionised water and stored at room temperature.

\section{Characterisation of hybrid nanoparticles}

Metal content analysis was carried out using inductively coupled plasma-optical emission spectroscopy (ICP-OES, Optima 7000V DV, PerkinElmer, Wokingham, UK). An acid digestion was carried out in conc. $\mathrm{HNO}_{3}: \mathrm{HCl}, 1: 5$ sample: acid). The samples were diluted with deionised water prior to analysis. A calibration was run using iron and gold standard solutions $0.5-5 \mathrm{mg} \mathrm{mL}^{-1}(R=0.9999)$. The concentration of HNPs used for all experiments indicates the concentration of Fe. Peak absorbance of HNPs in deionized water was measured using a UV-2600 UV-Vis (NIR) with an ISR-2600 Plus Integrated sphere (Shimadzu, Germany). Samples were analysed in quartz cuvettes, absorbance scans were carried out between 200-1200 nm. The hydrodynamic diameter and polydispersity index were estimated using photon correlation spectroscopy (PCS) (Zetasizer Nano-ZS, Malvern Instruments, Malvern, UK). All measurements were carried out at $25^{\circ} \mathrm{C}$ $(n=3)$ and an average value determined. Zeta potential measurements were carried out to determine surface charge using the same instrument throughout the synthetic pathway. Transmission electron microscopy (TEM) was used to visualize the particles. Samples were pipetted onto formvar coated copper grids $(2 \mu \mathrm{L})$ and dried under a heat lamp for 4 h. The grids were directly imaged using a JEOL JEM-1230 microscope with ANAlysis software (JEOL, Japan).

\section{Laser irradiation of HNPs}

HNPs $\left(500 \mu \mathrm{gmL}^{-1}, 50 \mu \mathrm{gmL}^{-1} 5 \mu \mathrm{gmL}^{-1}\right.$ and $\left.0 \mu \mathrm{gmL}^{-1}\right)$ were dispersed into a $2 \%$ agar phantom in $35 \mathrm{~mm}$ petri dishes (Greiner, UK). The samples were exposed to laser irradiation at $1064 \mathrm{~nm}$ using a ML-
A

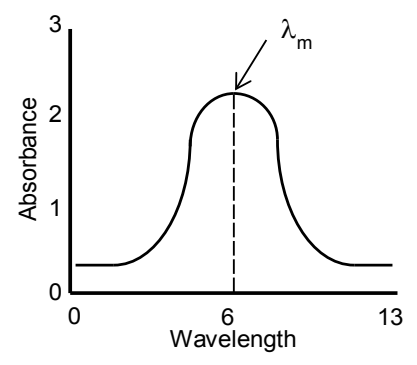

B

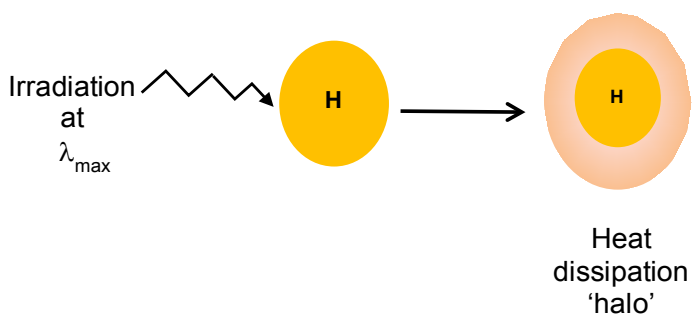

Figure 1: Exploitation of SPR for heating HNPs. A) Schematic representation of $\lambda_{\max }$ absorbance of gold nanoparticles resulting in optimal SPR wavelength and $B$ ) respresentation of heating pattern resulting from laser irradiation. 
LASER-YB5 Q-switched Nd:YAG Laser Treatment System (WeiFang MingLiang Electronics Company Ltd., China) Pulse width: $10 \mathrm{ns,}$ pulse repetition frequency: 1-6 Hz, laser spot diameter: $3 \mathrm{~mm}$, cooling system: water cooled with airflow cooling. The beam was collimated through concave lenses to a $1 \mathrm{~mm}$ diameter and passed through the gel centre. The gels were irradiated for 60 seconds and their cooling profile monitored over 120 seconds. Changing temperature in the samples was calculated as (Equation 1):

$$
\Delta \mathrm{T}=\left(\mathrm{T}_{\text {final }}-\mathrm{T}_{\text {initial }}\right)-\mathrm{T}_{\Delta \text { control }}
$$

\section{Thermal monitoring using thermocouples}

Temperature change was monitored using $0.076 \mathrm{~mm}$ diameter PFA coated T-type thermocouples (Omega, UK). One thermocouple was positioned at the centre of the laser spot and four others were placed at $1 \mathrm{~mm}, 2 \mathrm{~mm}, 4 \mathrm{~mm}$ and $8 \mathrm{~mm}$ distance from the irradiation point. All measurements were carried out at room temperature, $25^{\circ} \mathrm{C}\left( \pm 3^{\circ} \mathrm{C}\right)$ with one temperature sample per second recorded using a PICO USB TC-08 data logger (Omega, UK).

\section{Thermal monitoring using infrared camera imaging}

Temperature change was measured using an Optris PI640 Thermal Imaging Camera (Optris, Germany). The camera was positioned $5 \mathrm{~cm}$ away from the gel and focussed on the irradiation site. The data was recorded on Optris PI Connect software (Optris, Germany). Data samples were recorded every 0.1 second and data analysis was carried out offline using direct measurement of the .vga files collected.

\section{Results and Discussion}

The fabrication of the HNPs was monitored closely through zeta potential measurement (Figure $2 \mathrm{~A}$ ). The $\mathrm{Fe}_{3} \mathrm{O}_{4}$ possessed a negative surface charge of $-24.9 \mathrm{mV}$ due to the surface sulfate associations from the synthetic procedure. Subsequently the zeta potential shifted to $+47.5 \mathrm{mV}$ after coating of the $\mathrm{Fe}_{3} \mathrm{O}_{4}$ core with PEI due to the positive charge of the primary amines in its backbone. The $\mathrm{Fe}_{3} \mathrm{O}_{4}-\mathrm{PEI}-\mathrm{Au}_{\text {seed }}$ and HNPs exhibited a decrease in surface charge, due to the negativity of the gold atoms $(+33.9 \mathrm{mV}$ and $-4.3 \mathrm{mV}$ respectively). The morphology and size was visualised using transmission electron microscopy (TEM). The image (Figure $2 \mathrm{~B}$ ) shows that spherical particles of approximately $50 \mathrm{~nm}( \pm 5, \mathrm{n}=25)$ were formed. Inductively coupled plasma - optical emission spectroscopy (ICP-OES) was used to deduce the total iron and gold content of the NPs. The concentration of iron and gold were observed to be $2.653 \mathrm{mgmL}^{-1} \pm 0.155$ and $0.6827 \mathrm{mgmL}^{-1} \pm 0.0043$, respectively. The ratio of Fe:Au was approximately 4:1.
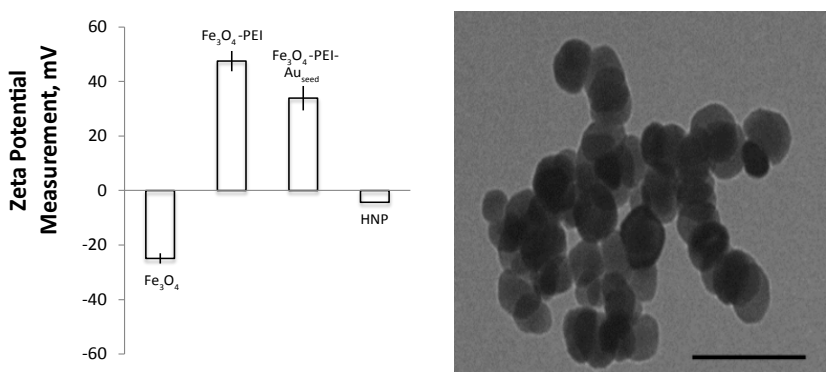

Figure 2: Physical properties of HNPs: A) Zeta potential monitoring of nanoparticles during synthetic procedure from iron oxide core to complete HNP structure. All measurements taken at $25^{\circ} \mathrm{C}(n=3, \pm S D)$. B) TEM image of HNPs.

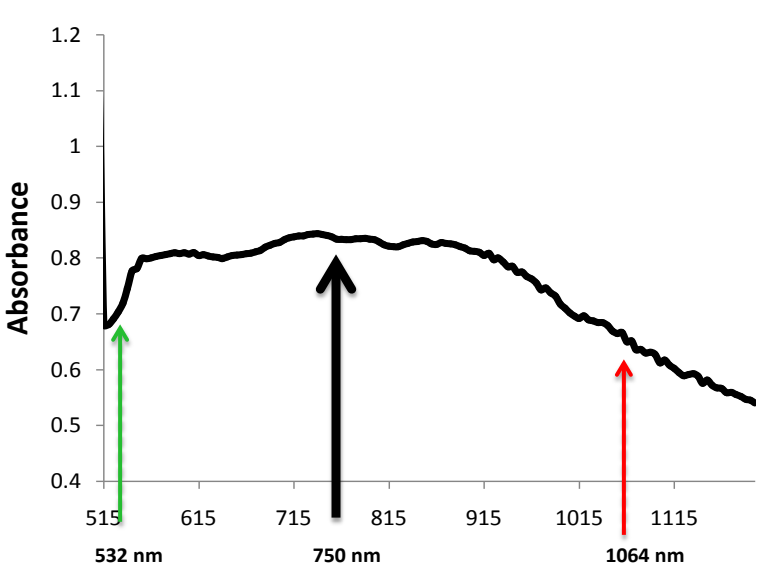

Wavelength, $\mathrm{nm}$

Figure 3: UV-Vis/NIR spectra of HNPs suspended in deionized water showing $\lambda_{\max }$ at $750 \mathrm{~nm}$ and absorbance at common irradiation wavelengths $532 \mathrm{~nm}$ and $1064 \mathrm{~nm}$.

Figure 3 shows the broad peak observed in the UV-Vis/NIR spectrum. The $\lambda_{\max }$ is observed at approximately $750 \mathrm{~nm}$ as indicated. This is expected as the maximum absorbance for gold nano-shells is larger than that of solid gold nanoparticles and fits with the architecture of the HNP. The broad peak covers a large array of wavelengths in which laser irradiation would become absorbed leading to heating effects in the HNP. It can be seen from the spectra that $532 \mathrm{~nm}$ does experience some absorbance which explains the rise in thermal energy reported in the literature for gold nano-shells after irradiation. As discussed, heating will occur at any point in the spectra where absorbance occurs due to the presence of gold. Towards the red end of the spectra at $1064 \mathrm{~nm}$ it is observed that comparable absorbance to $532 \mathrm{~nm}$ occurs. Therefore, it is postulated that laser irradiation at $1064 \mathrm{~nm}$ should result in localized heating of the HNPs without heating of biological tissues.

In order to test this theory, the HNPs were suspended in $2 \%$ agar phantom. Previous reports have suggested that this gel is a good mimic for biological tissue [27], although others also exist [28]. The nanoparticles were evenly suspended in the gels and allowed to cool to room temperature before use. Two methods of thermal measurement were used and compared for accuracy. The nanoparticles were irradiated for 60 seconds and their thermal change recorded over 240 seconds. The thermal change reported was in relation to a control sample of agar alone in order to take into consideration any absorption from the matrix, which was negligible. The data obtained from the thin wired thermocouples (Figure 4A) suggest that the degree of heating was related to the concentration of nanoparticles. The $500 \mu \mathrm{gmL}^{-1}$ sample experienced a $30^{\circ} \mathrm{C}$ temperature increase whilst the $5 \mu \mathrm{gmL}^{-1}$ and $50 \mu \mathrm{gmL}^{-1}$ samples experienced a $7^{\circ} \mathrm{C}$ and $25^{\circ} \mathrm{C}$ temperature increase, respectively. However, large standard deviations were evident between the experiments, which ultimately rendered no significant difference between the $50 \mu \mathrm{gmL}^{-1}$ and $500 \mu \mathrm{gmL}^{-1}$ samples $(\mathrm{p}<0.001)$. All samples had returned to ambient temperature after the 240 second period. As expected the sample with the lowest concentration $\left(5 \mu \mathrm{gmL}^{-1}\right)$ returned to ambient temperature quicker, after 115 seconds post irradiation. The $50 \mu \mathrm{gmL}^{-1}$ and $500 \mu \mathrm{gmL}^{-1}$ samples returned to ambient temperature after 135 seconds post irradiation, interestingly at the same time point.

The data obtained using the infrared camera in general followed 
A

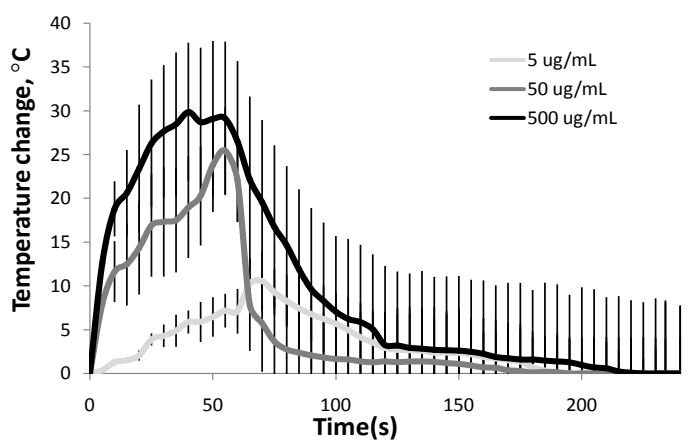

B

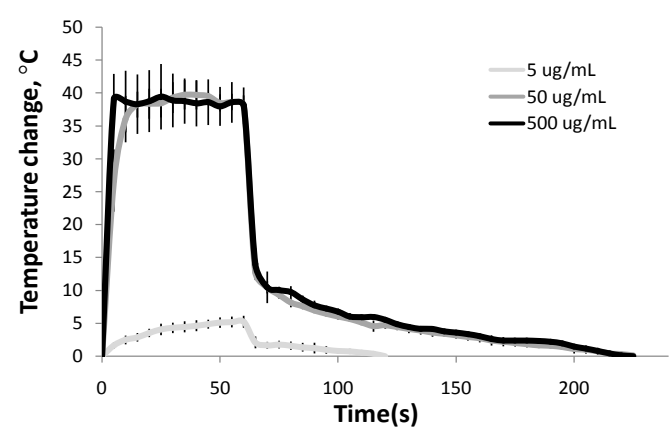

Figure 4: Temperature change and cooling profile of HNPs suspended in $2 \%$ agar phantom. Irradiation time fixed at 60 seconds. Temperature change measured using A) thin wire thermocouples and B) infrared camera. All temperature changes quoted in relation to control sample $(n=3, \pm S D)$.

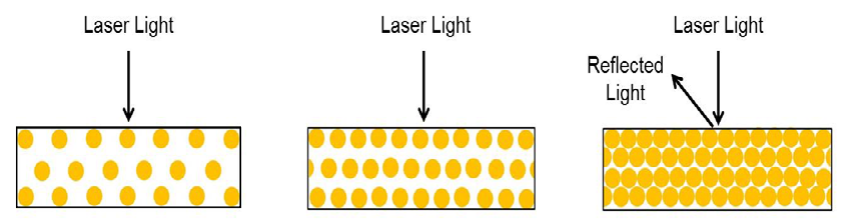

Increasing concentration of HNPs in agar phantom $\longrightarrow$

Figure 5: Schematic diagram detailing reduction in SPR potential experienced by HNPs at higher concentrations due to surface saturation in agar and resultant reflectance thus limiting heat generation.

a similar trend. Both the higher concentration samples $\left(50 \mu \mathrm{gmL}^{-1}\right.$ and $500 \mu \mathrm{gmL}^{-1}$ ) exhibited a thermal rise of $40^{\circ} \mathrm{C}$; at this temperature the graph plateaued indicating that this was the maximal heating achievable for these particles. Post-irradiation data was in agreement with the thermocouple data in that the samples returned to ambient temperature after 135 seconds. Both sets of data suggest that there is a threshold concentration whereby the HNPs have achieved maximal potential and no further increase of concentration would be beneficial. The rationale behind such a threshold is postulated to be due to the relative spacing of the HNPs within the phantom matrix. At any given temperature the NPs will be a certain distance apart (assuming even distribution). As these experiments are measuring bulk increase due to the cumulative effect of the HNPs present and not due to a single nanoparticulate, it would be rational to hypothesise that as the HNP concentration increases and distance between the particles decrease, the scope for SPR decreases. This is due to the surface saturation of the sample with the HNPs, whereby particles organise themselves across the surface much like surfactant molecules in water but once saturation is reached the subsequent particle must reside in the bulk media below. Hence, the HNPs on the surface of the media are suitably positioned for exposure to irradiation whilst the particles below are shielded. Also there is a possibility of laser beam reflectance as the phantom becomes more iridescent due the saturated matrix surface, resulting in reduced scope for absorbance and SPR (Figure 5).

In this study we have used concentrations as high as $500 \mu \mathrm{gmL}^{-1}$ for scientific interest, which are not indicative of the concentrations which would be used clinically. The lowest concentration tested $\left(5 \mu \mathrm{gmL}^{-1}\right)$ experienced a mild thermal increase in agreement with the thermocouple data, with a 5 degree temperature change observed. These findings show that even though the irradiating wavelength is not at the $\lambda_{\max }$ of the HNP UV absorbance, because it is within the broad peak of the spectra the particles are still capable of undergoing SPR and heating can be achieved. An additional study into the effect of this laser on tissue has been carried out by irradiation of chicken breast tissue and thermal monitoring using the infrared camera. This study showed than minimal $\left(<1^{\circ} \mathrm{C}\right)$ heating occurred at the laser culmination point with no heat dissipation observed at $1 \mathrm{~mm}$ distance after 120 seconds of laser irradiation (Supplementary Material, Figure A).

The heat dissipation through the agar phantom was measured using the two systems. It was assumed that since the HNPs were spherical that heat distribution was identical at all points of the spheres exterior resulting in a uniform halo like effect (Figure 1). The data obtained using the thin wired thermocouples (Figure 6A) showed that no heating effect was experienced $8 \mathrm{~mm}$ from the laser irradiation site. After 60 seconds irradiation all samples resulted in thermal rise at a distance of $4 \mathrm{~mm}$ from the laser site. Interestingly, after 30 seconds post irradiation the gel was still experiencing heating effects at this distance. At $1 \mathrm{~mm}$ distance from the irradiation site the gel temperature had increased by $3.29^{\circ} \mathrm{C}, 15.43^{\circ} \mathrm{C}$, and $14.81^{\circ} \mathrm{C}$ at $5 \mu g \mathrm{gL}^{-1}, 50 \mu \mathrm{gmL} \mathrm{L}^{-1}$ and $500 \mu \mathrm{gmL}^{-1}$, respectively. This was decreased to $2.91^{\circ} \mathrm{C}, 9.32^{\circ} \mathrm{C}$ and $8.21^{\circ} \mathrm{C}$ at $2 \mathrm{~mm}$ and $1.19^{\circ} \mathrm{C}, 3.65^{\circ} \mathrm{C}$ and $3.05^{\circ} \mathrm{C}$ at $4 \mathrm{~mm}$ (respectively).

The infrared imaging data for the heat dissipation study (Figure 6B) showed similar trends, whereby the data for the $50 \mu \mathrm{gmL}^{-1}$ and $500 \mu \mathrm{gmL}^{-1}$ samples was comparable. However, after 60 seconds irradiation these higher concentration samples resulted in heating effects as far as $6 \mathrm{~mm}$ away from the laser spot. At $1 \mathrm{~mm}$ distance from the irradiation site the gel temperature had increased by $5.03^{\circ} \mathrm{C}$, $36.06^{\circ} \mathrm{C}$, and $27.1^{\circ} \mathrm{C}$ at $5 \mu \mathrm{gmL}^{-1}, 50 \mu \mathrm{gmL}^{-1}$ and $500 \mu \mathrm{gmL}^{-1}$ respectively. This was decreased to $5.13^{\circ} \mathrm{C}, 26.60^{\circ} \mathrm{C}$ and $21.37^{\circ} \mathrm{C}$ at $2 \mathrm{~mm}$ and $1.83^{\circ} \mathrm{C}$, $27.13^{\circ} \mathrm{C}$ and $10.10^{\circ} \mathrm{C}$ at $4 \mathrm{~mm}$ (respectively).

The major differences in the reliability of these measurement techniques can be clearly identified from the Figures 4 and 6 . The thin wired thermocouple data possessed notably higher standard deviations than the infrared camera measurements. Figure $6 \mathrm{~B}$ has relatively small standard deviations (compared with Figure 6A) and in common with the heating experiments, large standard deviations were observed in the heat dissipation studies using the thermocouples. Additionally, the infrared imaging allowed for increased data points and we believe more sensitive and robust data in comparison with the thin wired thermocouples. The infrared imaging also allows for visual demonstration of the thermal changes through the heat maps generated (Figure 7), which can show dissipation more effectively via color scale, which can often be achieved graphically, as well as time lapse videos. Figure 6 shows the heat map of all three concentrations of HNP after 60 seconds. At the higher concentrations, a visible 'halo' can be seen which shows the heat dissipating out towards the exterior surroundings of the NPs. These visual representations can be useful 
A

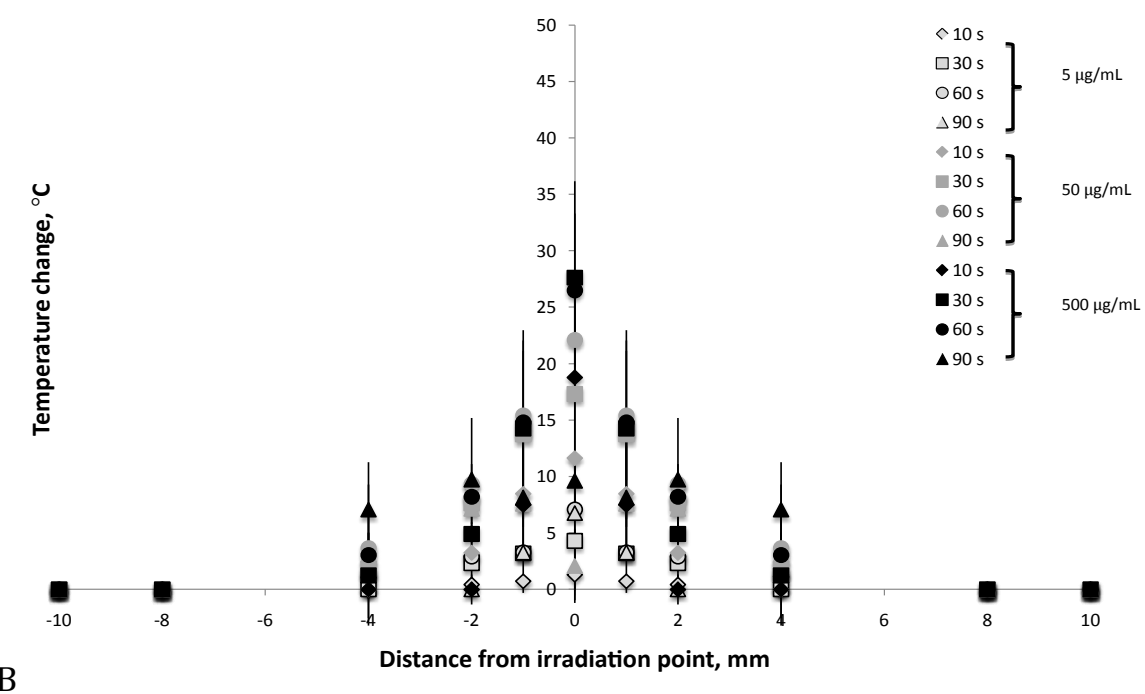

B

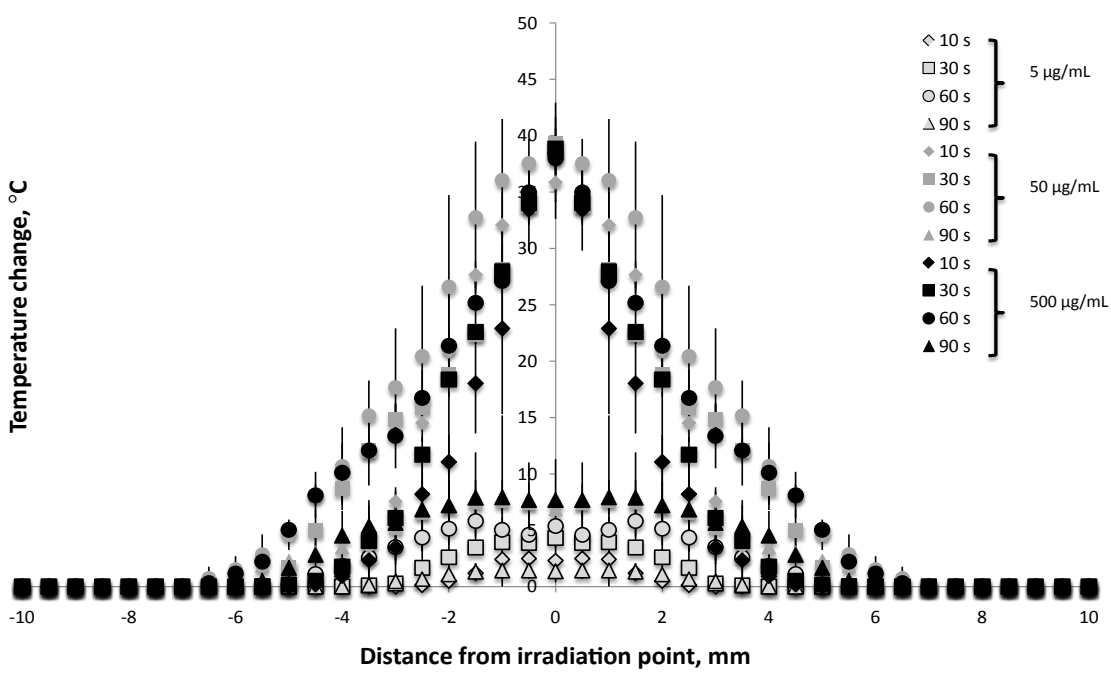

Figure 6: Thermal dissipation in HNP samples measured from irradiation point. Measurements gathered using A) thin wire thermocouples and B) infrared camera. All thermal change stated in relation to control sample ( $n=3, \pm S D)$.

for modelling in order to mathematically determine the fate of these HNPs after laser irradiation at any given concentration. These studies are ongoing in our laboratory in order to elucidate the rationale behind the threshold concentration at which no further heating is observed.

The major knowledge gap in use of HNPs for thermal applications such as for ablation or thermal responsive switches for drug delivery is the heat generation and dissipation ability. These studies have indicated the HNPs were capable of achieving thermal rise as high as $40^{\circ} \mathrm{C}$ from ambient temperature. This indicates that pulsed irradiation using this relatively inexpensive laser system at $1064 \mathrm{~nm}$ is a viable option for translation of these particles into the clinic. In order for thermal ablation to occur, cells or tumour tissue need to be heated up to temperatures where irreversible stress is caused to the cell resulting in mortality. Biological systems are relatively fragile and, as such a $40^{\circ} \mathrm{C}$ increase from body temperature (assuming cancerous tissue exists at an increased temperature of $42^{\circ} \mathrm{C}$ ) up to $82^{\circ} \mathrm{C}$ using $50 \mu \mathrm{gmL}^{-1}$ would cause a dramatic effect and certainly result in tissue damage beyond
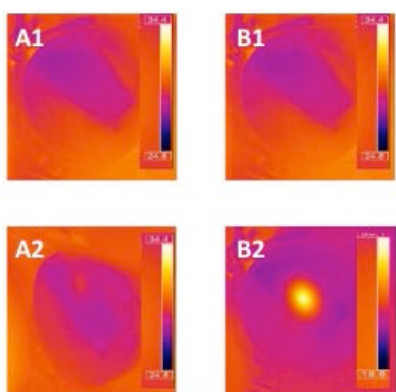
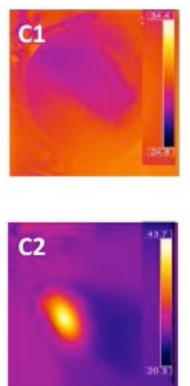

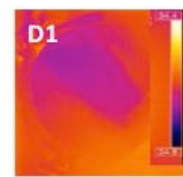

D2

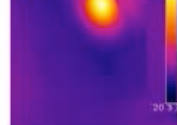

Figure 7: Heat dissipation maps of HNP gels irradiated for 60 seconds. Data collected using an infrared camera.

repair. The interesting findings from this work are that whilst many reports suggest gold nanoparticulates result in highly localised heating, our samples experienced thermal rise in the phantom up to $6 \mathrm{~mm}$ from 
the laser source. This means that a total area of $132.73 \mathrm{~mm}^{2}$ experienced heating, which is actually quite a substantial area. This would need to be taken into account in relation to tumour size or total irradiation area required. Although, at the $6 \mathrm{~mm}$ distance the heating rise from the $42^{\circ} \mathrm{C}$ of cancer tissue would amount to less than $50^{\circ} \mathrm{C}$ and hence is unlikely to have lasting effects, at $4 \mathrm{~mm}$ the total heat rise would be above $10^{\circ} \mathrm{C}$ and would result in irreversible adverse effects.

The alternative use for HNPs to act as thermal triggers for targeted drug delivery comes with different challenges. Firstly, the drugs are required to be conjugated onto the HNP surface via thermally labile linkers. It is important to remember that these linkers can often consist of long chains or polymers, which may exist in conformations such that they reside at some distance from the particle surface. Therefore, the heat dissipation from the HNP needs to be such that the thermally labile linker experiences the temperature increase for payload release, otherwise the system would be rendered useless. Additionally, the heating effects must be precise and controllable, in that the thermal rise must be adequate for drug release but not for cellular stress or damage. Our findings suggest that the lowest concentration sample would be ideal for such application. Not only was the maximal heat change experienced $\left(7^{\circ} \mathrm{C}\right)$ which would result in tissue heating to $49^{\circ} \mathrm{C}$, but this thermal rise appeared to be maintained over the duration of irradiation, indicating control could be achieved (Figure 5B). Additionally, the heat dissipation observed was at a maximal distance of $4 \mathrm{~mm}$, which would result in an area of $63.62 \mathrm{~mm}^{2}$ being affected. At this low concentration, the area affected is less of an issue due to the mild heating effects $\left(<50^{\circ} \mathrm{C}\right)$, which should not cause any irreversible negative effects on the surrounding tissue. The heat dissipation data is also encouraging as it ensures that any linker which would be a distance of Angstroms away from the particle surface would definitely experience the thermal rise resulting in payload release.

These findings highlight the exciting potential for these systems to be used as thermo-responsive carriers in cancer therapy. The benefits of the magnetic core arising from the iron oxide also allow for image guidance, which would be beneficial to ensure targeted irradiation. Further work is ongoing in our laboratory in order to further evaluate the potential of these HNPs biologically by irradiation in vitro and monitoring cellular response.

\section{Conclusion}

This study highlights the potential of HNPs as thermal switches in heat triggered drug delivery. Although the SPR optimal wavelength of the particles was $750 \mathrm{~nm}$, our HNPs observed heat generation up to $40^{\circ} \mathrm{C}$ and dissipation up to $6 \mathrm{~mm}$ away from radiation site using a $1064 \mathrm{~nm}$ pulsed Nd:YAG laser. Additionally the HNPs may also be employed in tumour ablation however due care is required in order to minimize heat dissipation to healthy tissues.

\section{Acknowledgement}

This work was funded by and carried out in the Institute for Science and Technology in Medicine, Keele University.

\section{References}

1. Kanazaki K, Sano K, Makino A, Shimizu Y, Yamauchi F, et al. (2015) Development of Anti-HER2 fragment antibody conjugated to iron oxide nanoparticles for in vivo HER2-targeted photoacoustic tumor imaging. Nanomed 11: 2051-2060.

2. Banu H, Sethi DK, Edgar A, Shweiff A, Raynees N, et al. (2015) Doxorubicin loaded polymeric gold nanoparticles targeted to human folate receptor upon laser photothermal therapy potentiates chemotherapy in breast cancer cell lines. J Photochem Photobiol B: Biol 149:116-128.
3. Liu J, Hu R, Liu J, Zhang B, Wang Y, et al. (2015) Cytotoxicity assessment of functionalized CdSe, CdTe and InP quantum dots in two human cancer cell models. Mater Sci Eng C Mater Biol Appl 57: 222-231.

4. Chou HT, Wang TP, Lee CY, Tai NH, Chang HY (2013) Photothermal effects of multi-walled carbon nanotubes on the viability of BT-474 cancer cells. Mater Sci Eng C Mater Biol Appl 33: 989-995.

5. Castiglioni S, Cazzaniga A, Perrotta C, Maier JA (2015) Silver nanoparticlesinduced cytotoxicity requires ERK activation in human bladder carcinoma cells. Toxicol Lett 237: 237-243.

6. Malekigorji M, Hoskins C, Curtis T, Varbiro G (2014) Enhancement of the cyctotoxic effect of anticancer agent by cytochrome $c$ functionalized hybrid nanoparticles in hepatocellular cancer cells. J Nanomed Res 1: 0010.

7. Khan MS, Vishakante GD, Siddaramaiah H (2013) Gold nanoparticles: paradigm shift in biomedical applications. Adv Colloid Interface Sci 199-200: $44-58$.

8. Jain PK, El-Sayed IH, El-Sayed MA (2007) Au nanoparticles target cancer, Nano Today 2: 18-29.

9. Jing L, Liang X, Deng Z, Feng S, Li X, et al. (2014) Prussian blue coated gold nanoparticles for simultaneous photoacoustic/CT bimodal imaging and photothermal ablation of cancer. Biomaterials 35: 5814-5821.

10. Lee HJ, Liu Y, Zhao J, Zhou M, Bouchard RR, et al. (2013) In vitro and in vivo mapping of drug release after laser ablation thermal therapy with doxorubicinloaded hollow gold nanoshells using fluorescence and photoacoustic imaging J Control Release 172: 152-158.

11. Liang XJ, Chen C, Zhao Y, Wang PC (2010) Circumventing tumor resistance to chemotherapy by nanotechnology. Methods Mol Biol 596: 467-488.

12. Chu KF, Dupuy DE (2014) Thermal ablation of tumours: biological mechanisms and advances in therapy. Nat Rev Cancer 14: 199-208.

13. Hines-Peralta A, Goldberg SN (2004) Review of radiofrequency ablation for renal cell carcinoma. Clin Cancer Res 10: 6328S-34S.

14. Woods $E$ (2010) Laser ablation of the prostate: a safe effective treatment of obstructive benign prostatic disease. Can Urol Assoc J 4: 344-346.

15. Cai W, Gao T, Hong $H$, Sun J (2008) Applications of gold nanoparticles in cancer nanotechnology. Nano Sci App 1:10,2147.

16. Clare H, Lin PK, Tetley L, Cheng WP (2012) The use of nano polymeric selfassemblies based on novel amphiphilic polymers for oral hydrophobic drug delivery. Pharm Res 29: 782-794

17. Moses MA, Brem H, Langer R (2003) Advancing the field of drug delivery: taking aim at cancer. Cancer Cell 4: 337-341.

18. Liu C, Li BQ, Mi CC (2009) Fast transient thermal analysis of gold nanoparticles in tissue-like medium. IEEE Trans Nanobioscience 8: 271-280.

19. Cheong SK, Krishnan S, Cho SH (2009) Modeling of plasmonic heating from individual gold nanoshells for near-infrared laser-induced thermal therapy. Med Phys 36: 4664-4671.

20. Jalani G, Naccache R, Rosenzweig DH, Lerouge S, Haglund L, et al. (2015) Real-time, non-invasive monitoring of hydrogel degradation using LiYF4:Yb(3+)/ Tm(3+) NIR-to-NIR upconverting nanoparticles. Nanoscale 7: 11255-11262.

21. Pissuwan D, Valenzuela SM, Cortie MB (2006) Therapeutic possibilities of plasmonically heated gold nanoparticles. Trends Biotechnol 24: 62-67.

22. Huang X, El-Sayed MA (2010) Gold nanoparticles: Optical properties and implementations in cancer diagnosis and photothermal therapy. J Adv Res 1:13-28

23. Tanaka Y, Matsuo K, Yuzuriha S (2011) Objective assessment of skin rejuvenation using near-infrared 1064-nm neodymium: YAG laser in Asians. Clin Cosmet Investig Dermatol 4: 123-130.

24. Lukianova-Hleb EY, Volkov AN, Wu X, Lapotko DO (2013) Transient enhancement and spectral narrowing of the photothermal effect of plasmonic nanoparticles under pulsed excitation. Adv Mater 25: 772-776.

25. Andres Verges M, Costo R, Roca AG, Marco JP, et al. (29008) Uniform and water stable magnetite nanoparticles with diameters around the mon- odomainmultidomain limit. J Phys D: Appl Phys 41: 134003. 
Citation: Curtis A, Malekigorji M, Holman J, Skidmore M, Hoskins C (2015) Heat Dissipation of Hybrid Iron Oxide-Gold Nanoparticles in an Agar Phantom. J Nanomed Nanotechnol 6: 335. doi:10.4172/2157-7439.1000335

26. Barnett C, Gueorguieva M, Lees M, McGarvey D, Hoskins C (2012) Effect of hybrid composition on physicochemical properties and morphology of iron oxide-gold nanoparticles. J Nano Res 14:1170.

27. Li C, Huang Z, Wang RK (2011) Elastic properties of soft tissue-mimicking phantoms assessed by combined use of laser ultrasonic and low coherence interferometry. Optics Express 9:10153-10163.

28. Lazebnik M, Madsen EL, Frank GR, Hagness SC (2005) Tissue-mimicking phantom materials for narrowband and ultrawideband microwave applications. Phys Med Biol 50: 4245-4258. 